\title{
RECURRENCE OF THYMOMA: ANALYSIS OF CLINICOPATHOLOGIC FEATURES, TREATMENT, AND OUTCOME
}

Enrico Ruffini, MD ${ }^{a}$

Maurizio Mancuso, $\mathrm{MD}^{\mathrm{a}}$

Alberto Oliaro, $\mathrm{MD}^{\mathrm{a}}$

Caterina Casadio, $\mathrm{MD}^{\mathrm{a}}$

Antonio Cavallo, $\mathrm{MD}^{\mathrm{a}}$

Roberto Cianci, $\mathrm{MD}^{\mathrm{a}}$

Pier Luigi Filosso, $\mathrm{MD}^{\mathrm{a}}$

Massimo Molinatti, $\mathrm{MD}^{\mathrm{a}}$

Calogero Porrello, $\mathrm{MD}^{\mathrm{a}}$

Nazario Cappello, $\mathrm{PhD}^{\mathrm{b}}$

Giuliano Maggi, $\mathrm{MD}^{\mathrm{a}}$
Objective and methods: This study reports clinicopathologic features, treatment, and outcome of 30 recurrent thymomas out of 266 totally resected thymomas. Results: The mean disease-free interval to recurrence was 86 months. Recurrence occurred less frequently and after a longer disease-free interval after resection of encapsulated versus invasive thymomas. The presence of associated myasthenia gravis did not affect recurrence proportion, disease-free interval, or survival after recurrence. A local recurrence occurred in 11 patients, 17 patients had a distant recurrence, and the extent of the recurrence could not be determined in 2 cases. Surgical treatment of the recurrent tumor was attempted in 16 cases, and a total resection was possible in 10 cases; exclusive radiotherapy was done in 11 cases. Overall 5and 10-year survivals were $48 \%$ and $24 \%$, respectively. In a univariate analysis, survival was significantly better in the presence of a local recurrence and in case of a total resection of the recurrent tumor. The use of adjuvant therapy after the resection of the initial thymoma had no effect on reducing the incidence of recurrence, in prolonging the disease-free interval, or in improving survival after the development of the recurrence. In a multivariate survival analysis, significant prognostic factors were the presence of a local recurrence and total resection of the recurrent tumor. Conclusions: Surgical resection is recommended in patients with recurrent thymoma. Local recurrence and total resection of the recurrent tumor are associated with excellent prognosis. A poor prognosis may be anticipated in the presence of distant recurrence and when radical surgical treatment is not done. (J Thorac Cardiovasc Surg 1997;113:55-63)
Surgical resection represents the main therapeutic

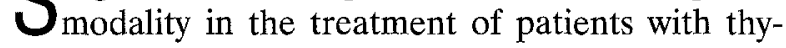
moma and has remarkable effects on long-term survival. Many authors have emphasized the beneficial impact on prognosis of postoperative radiotherapy, ${ }^{1-4}$ which in several studies was found to improve survival after resection of invasive thymomas. Others have advocated the use of preoperative chemotherapy in advanced invasive thymomas, and

From the Department of Thoracic Surgery a and the Department of Genetics, Section of Biostatistics, ${ }^{b}$ University of Torino, Torino, Italy.

Received for publication Oct. 10, 1995; revisions requested Jan. 4, 1996; revisions received August 7, 1996; accepted for publication August 7, 1996.

Address for reprints: Giuliano Maggi, MD, Department of Thoracic Surgery, University of Torino, 39/1, Via Millefonti, 10126 Torino, Italy.

Copyright (C 1997 by Mosby-Year Book, Inc.

$0022-5223 / 97 \$ 5.00+0 \quad 12 / 1 / 77141$ in one series this was found to yield encouraging results on survival. ${ }^{5}$

Unfortunately, the natural history of thymoma remains unpredictable because of the possibility of recurrence of the tumor at follow-up. Most authors agree that a prolonged follow-up is necessary in patients who undergo resection of thymoma because recurrence may occur up to several years after operation. ${ }^{1,6-8}$ The recurrence is generally confined to the mediastinum, and sometimes intrathoracic pleural dissemination may occur, whereas distant metastases are rare.

Recurrence is not infrequent after resection of either encapsulated thymoma or invasive tumors. The possibility that a thymoma may recur after total resection of the initial thymoma has been frequently reported in the literature. ${ }^{6,8,9}$ Controversy exists concerning optimal treatment of the recurrent tumor. Both proponents of operation and proponents of radiotherapy have reported interesting, although conflicting, results. 
Table I. Postsurgical staging of thymoma

\begin{tabular}{ll}
\hline Stage & \multicolumn{1}{c}{ Definition } \\
\hline I & Macroscopically, completely encapsulated; microscopically, no capsular invasion \\
II & Macroscopic invasion in surrounding fatty tissue or mediastinal pleura; microscopic invasion into the capsule \\
III & Macroscopic invasion into a neighboring organ, such as pericardium, great vessels, or lung \\
IVA & Pleural or pericardial dissemination \\
IVB & Hematogenous or lymphogenous metastases \\
\hline
\end{tabular}

Table II. Recurrence proportions by surgical-pathologic staging and by use of adjuvant radiotherapy after resection of initial thymoma

\begin{tabular}{lccc}
\hline \multicolumn{1}{c}{ Resection } & $\begin{array}{c}\text { Recurrence proportion } \\
\text { after initial resection }\end{array}$ & $\begin{array}{c}\text { Recurrence proportion } \\
\text { after adjuvant therapy } \\
\text { and resection }\end{array}$ & $\begin{array}{c}\text { Recurrence proportion } \\
\text { with no adjuvant } \\
\text { therapy after resection }\end{array}$ \\
\hline Encapsulated (stage I) & $7 / 152(5 \%)$ & $0 / 7(0 \%)$ & $7 / 145(5 \%)$ \\
Stage II & $6 / 58(10 \%)$ & $4 / 13(31 \%) *$ & $2 / 45(4 \%)$ \\
Stage III & $15 / 50(30 \%)$ & $9 / 14(64 \%)^{*}$ & $6 / 36(16 \%)$ \\
Stage IVa & $2 / 6(33 \%)$ & $1 / 2(50 \%)$ & $1 / 4(25 \%)$ \\
Invasive (total) & $23 / 114(20 \%) \dagger$ & $14 / 29(48 \%) \%$ & $9 / 85(10 \%)$ \\
\hline
\end{tabular}

${ }^{{ }^{*} p}=0.02$ versus corresponding stage/no adjuvant therapy.

$\dagger p=0.001$ versus encapsulated thymomas.

$\$ p=0.0002$ versus invasive/no adjuvant therapy.

Table III. Recurrence proportions by surgicalpathologic staging in patient population and separately in $M G$ and non-MG recurrent thymomas

\begin{tabular}{lccl}
\hline Stage & Total & $\begin{array}{c}\text { Myasthenic } \\
\text { thymomas }\end{array}$ & $\begin{array}{c}\text { Nonmyasthenic } \\
\text { thymomas }\end{array}$ \\
\hline I & $7 / 152(5 \%)$ & $5 / 99(5 \%)$ & $2 / 53(4 \%)$ \\
II & $6 / 58(10 \%)$ & $5 / 45(11 \%)$ & $1 / 13(8 \%)$ \\
III & $15 / 50(30 \%)$ & $11 / 35(31 \%)$ & $4 / 15(26 \%)$ \\
IVa & $2 / 6(33 \%)$ & $1 / 2(50 \%)$ & $1 / 4(25 \%)$ \\
$\quad$ Total & $30 / 266(11 \%)$ & $22 / 171(13 \%)^{*}$ & $8 / 95(8 \%)$ \\
\hline
\end{tabular}

${ }^{*} p=0.2$ versus nonmyasthenic thymomas.

The aim of the present study was to analyze cases of recurrent thymoma after a total resection of the tumor in the patient population seen at our department to evaluate the frequency of recurrence, disease-free interval to recurrence, treatment modalities, and outcome of these patients in relation to clinicopathologic features of the initial thymoma, associated myasthenia gravis (MG), and the total population of patients with nonrecurrent thymoma.

\section{Patients and methods}

This study was undertaken under our Investigational Review Board's guidelines for retrospective chart review.

Between 1974 and 1993 a total of 310 thymomas were operated on at the Department of Thoracic Surgery of the University of Torino. Among the patients, 266 received a total resection of the tumor. Thirty patients out of these 266 had a recurrence of the initial thymoma, and they represent the population of the present study. There were 16 men and 14 women with a mean age at the time of the original operation of 42 years (range 20 to 71 years). Surgical-pathologic staging of the initial thymoma according to Masaoka and colleagues ${ }^{10}$ was done (Table I) and revealed 7 encapsulated (stage I) and 23 invasive (6 stage II, 15 stage III, and 2 stage IVa) thymomas. MG was present in 22 patients. Surgical intervention for all patients with and without MG included resection of the thymoma and thymectomy. Histologic study revealed 22 mixed lymphoepithelial, 4 predominantly epithelial, and 3 predominantly lymphocytic thymomas and 1 thymic carcinoma.

The surgical approach was sternotomy in 22 cases, posterolateral thoracotomy in 3 cases, and cervicotomy with sternal split in 5 cases. Postoperative radiation therapy was offered to patients with invasive thymoma. Radiotherapy was administered with ${ }^{60} \mathrm{Co}$ or $18 \mathrm{MeV}$ photons from a linear accelerator. Doses ranged between 38 and $44 \mathrm{~Gy}$ in fractions of 1.8 to 2 Gy over 4 to 5 weeks. Overall, 14 patients with recurrent thymoma received adjuvant radiotherapy after resection of the initial thymoma, including 4 with stage II, 9 with stage III, and 1 with stage IVa disease. In 16 patients no adjuvant therapy was used after operation because of the tumor staging (encapsulated thymomas in 7 cases), patient refusal, transfer of patient to another institution, or other reasons. The interval from thymectomy to the recurrence (disease-free interval) was defined as the period from the operation for the initial thymoma to the diagnosis of recurrence.

The extent of the recurrence was evaluated by the classification of Masaoka and associaties ${ }^{10}$ (Table I). One patient had a stage I recurrence, and 1,9, 13, and 4 patients had stage II, stage III, stage IVa, and stage IVb recurrences, respectively. In 2 patients the extent of the 


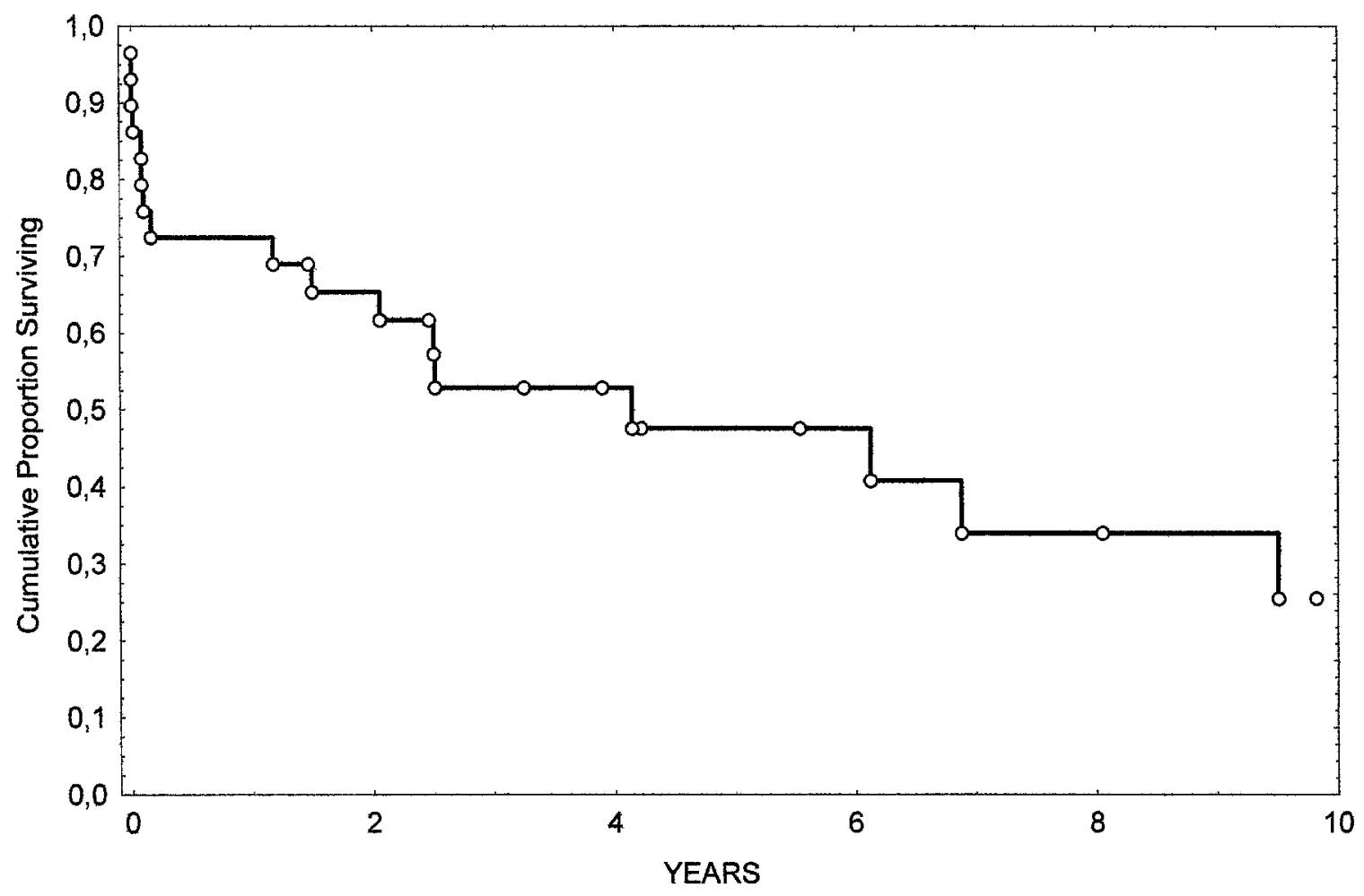

Fig. 1. Overall survival in the 30 cases of recurrent thymoma from the time of recurrence to death or most recent follow-up.

recurrence was uncertain and thus was not defined. Stage I through III recurrences were considered as local recurrences, whereas stages IVa and IVb were considered distant recurrences. By this classification, 11 patients had a local recurrence and 17 a distant recurrence.

The treatment of the recurrent tumor varied according to the extent of the recurrence, the control of $\mathrm{MG}$, and the general condition of the patient. Operation was done in 16 cases; radiotherapy was used in 11 cases and of these, 7 had adjuvant radiotherapy after the original operation. In 3 patients information regarding recurrence therapy was lacking. Almost always, recurrent thymomas were approached by a total longitudinal sternotomy. In only a minority of cases, when the recurrence was in the form of unilateral pleural dissemination without mediastinal involvement, a posterolateral thoracotomy was preferred. Occasionally, an additional thoracotomic access done one or two intercostal spaces below the first one (using the same skin incision) was necessary to properly resect recurrent lesions on the diaphragm, with a technique similar to that used in pleural mesothelioma.

A presumably total resection of the recurrent tumor was obtained in 10 cases. Of these, 8 had a local recurrence and 2 a distant recurrence. A subtotal resection with debulking intent was undertaken in 6 patients because of the extent of the recurrence. Radiotherapy was used as definitive treatment of the recurrence in 11 cases. Of these, 2 had a local recurrence and 9 a distant recurrence.

The statistical significance of recurrence proportions was tested with $2 \times 2$ contingency tables. Student's $t$ test (with Fisher's exact test when appropriate) was used to assess significance levels for variables measured on an interval scale. A probability value less than $5 \%(p<0.05)$ was regarded as significant. Survival rates were computed by the Kaplan-Meier method; the log-rank test was used to compare survival curves. A multivariate analysis was done by the stepwise proportional-hazards method of Cox. ${ }^{11}$ Data were analyzed with the use of STATISTICA release 5.0 software (StatSoft, Tulsa, Okla.).

\section{Results}

Recurrence proportions. Among 266 radically resected thymomas at our institution, 30 recurred (11\%, 30/266). Recurrence after initial resection of encapsulated thymomas occurred less frequently than recurrence after initial resection of invasive thymomas (Table II). The use of adjuvant therapy after complete resection of invasive thymomas was not associated with a decrease of the incidence of recurrence, either overall or by stage (a statistical analysis by stage was possible only for stage II and stage III thymomas); in fact, adjuvant radiotherapy was associated with an increase of recurrences (Table II). The recurrence proportion of patients with 


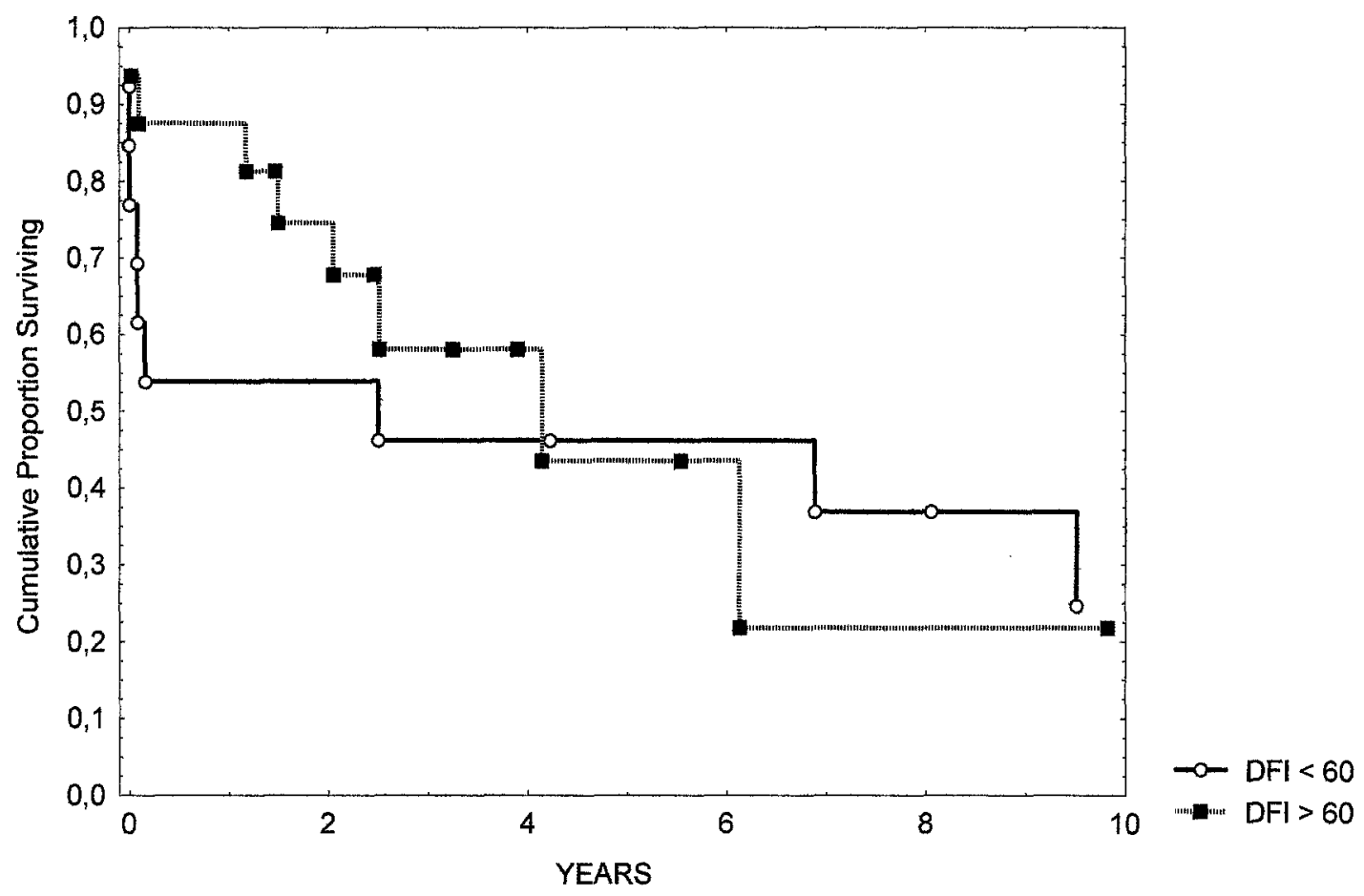

Fig. 2. Survival by the disease-free interval. $D F I<60$, Disease-free interval shorter than 60 months; $D F I$ $>60$, disease-free interval longer than 60 months; $p=0.7$.

and without associated MG did not show statistically significant differences (Table III) either overall or by stage.

Disease-free interval. Analysis of disease-free interval was done on the population of 30 patients with recurrent thymomas. Disease-free interval ranged from 4 to 192 months (mean 86, standard deviation 45 months; the variable follows a normallike distribution). A longer mean disease-free interval was found in encapsulated thymomas than in invasive thymomas (132 vs 71 months, $p=0.002$ ). The use of adjuvant therapy had no effect on prolonging the disease-free interval in invasive thymomas. In fact, mean disease-free interval was longer in patients who did not receive adjuvant therapy than in those who did (110 vs 57 months, $p=0.04$ ).

Extent of recurrence. The extent of recurrence was analyzed with regard to the stage of the initial thymoma and separately for MG and non-MG recurrent thymomas. We found that the incidence of recurrence in patients with MG was the same as that in the overall group of patients. Both local and distant recurrences were observed after initial thymomas in every stage, although a local recurrence was slightly more frequent in earlier stages of the initial thymoma and in MG thymomas. The small number of cases, however, did not allow statistical analysis. Overall, a local recurrence occurred in 2 $(33 \%)$ of 6 cases of non-MG recurrent thymomas (in 2 cases the extent of the recurrence was not determined) and in $9(41 \%)$ of 22 cases of $M G$ recurrent thymomas.

Survival. Survival curves were calculated from the time of recurrence to the event of interest (death or the most recent follow-up in censored observations). The time from the initial operation to recurrence was considered as a covariate in the analysis. Overall 5- and 10-year survivals for the 30 recurrent thymomas were $48 \%$ and $24 \%$ (Fig. 1).

We performed a survival analysis by the KaplanMeier method, adjusting for each of five independent variables (Figs. 2 to 6): (1) disease-free interval (>60 or $<60$ months); (2) MG; (3) adjuvant therapy after resection of the initial thymoma; (4) treatment of the recurrent tumor (complete resection, subtotal resection, nonsurgical therapy); and (5) extent of the recurrence (local or distant). We compared the survival curves with use of the log-rank test. Survival was significantly better in patients who did not 


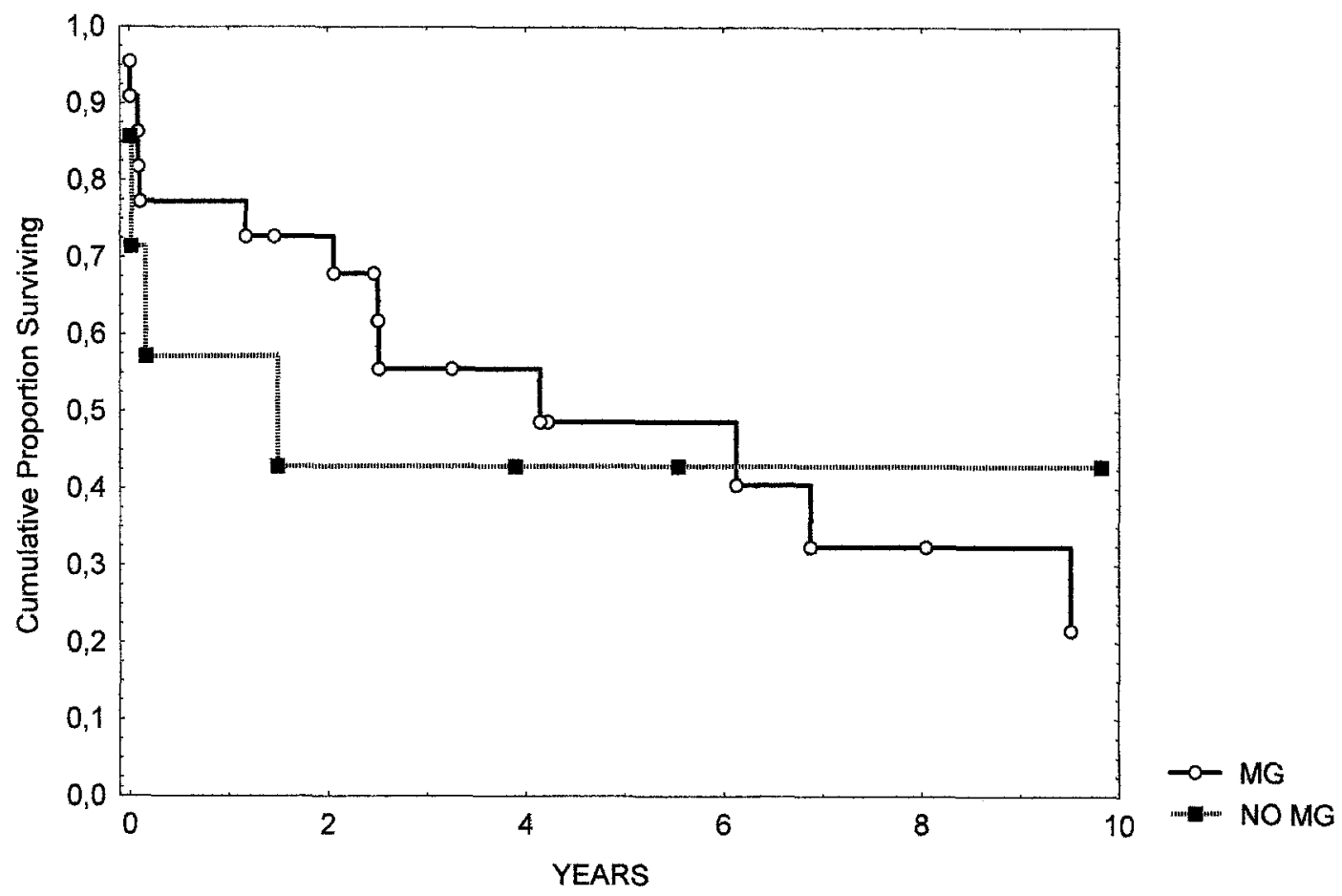

Fig. 3. Survival by the presence of associated MG; $p=0.8$.

receive adjuvant therapy $(p=0.04)$, in patients in whom the recurrent tumor could be totally resected $(p=0.008)$, and in patients with a local recurrence $(p=0.004)$. By contrast, the presence or absence of associated $\mathrm{MG}$ and a disease-free interval shorter or longer than 60 months had no influence on survival.

A Cox regression model was used adjusting for all the aforementioned independent variables (diseasefree interval was considered in the multivariate analysis as a continuous rather than a categorical variable) and adjusting for age considering two groups of patients, younger and older than 40 years, which approximates the mean age of the patient population (the variable follows a normal-like distribution). A final model was selected that included only those variables that were significant. Significant independent prognostic factors were the extent of the recurrence $\left(\beta=1.84 ; \mathrm{SE}^{*} 0.88 ; p=0.03\right)$, total resection of the recurrent tumor $(\beta=1.85 ; \mathrm{SE}=$ $0.84 ; p=0.03$ ), and absence of adjuvant therapy after the initial resection $(\beta=-1.44$; $\mathrm{SE}=0.69$; $p=0.04)$.

\footnotetext{
*Standard error.
}

\section{Discussion}

Operation represents the main therapeutic modality in patients with thymoma, with the possible use of adjuvant radiotherapy or chemotherapy. Complete resection of the thymoma has been found to have a remarkable effect on the long-term survival of these patients. Unfortunately, the possibility of recurrence after total resection of the tumor exists and has been extensively reported in the literature. A number of reports indicate recurrence rates after total resection of thymoma variably ranging from $8 \%$ to $18 \% .^{3,6,9}$ Our proportion of $11 \%$ of 226 total resections compares favorably with the results in the literature. Recurrence rates are correlated with the clinical stage. After resection of encapsulated thymomas a modest recurrence rate may be anticipated, which has been variably reported in the literature between $0 \%$ and $10 \%$. $3,7,12$ The corresponding figure in the present series was $5 \%(7 / 152)$. The recurrence proportion after resection of invasive thymomas in the present series was $20 \%$ (23/114), and comparable figures ranging from $11 \%$ to $36 \%$ have been reported by several investigators. $3,7,12,13$

The association of thymoma and MG has been 


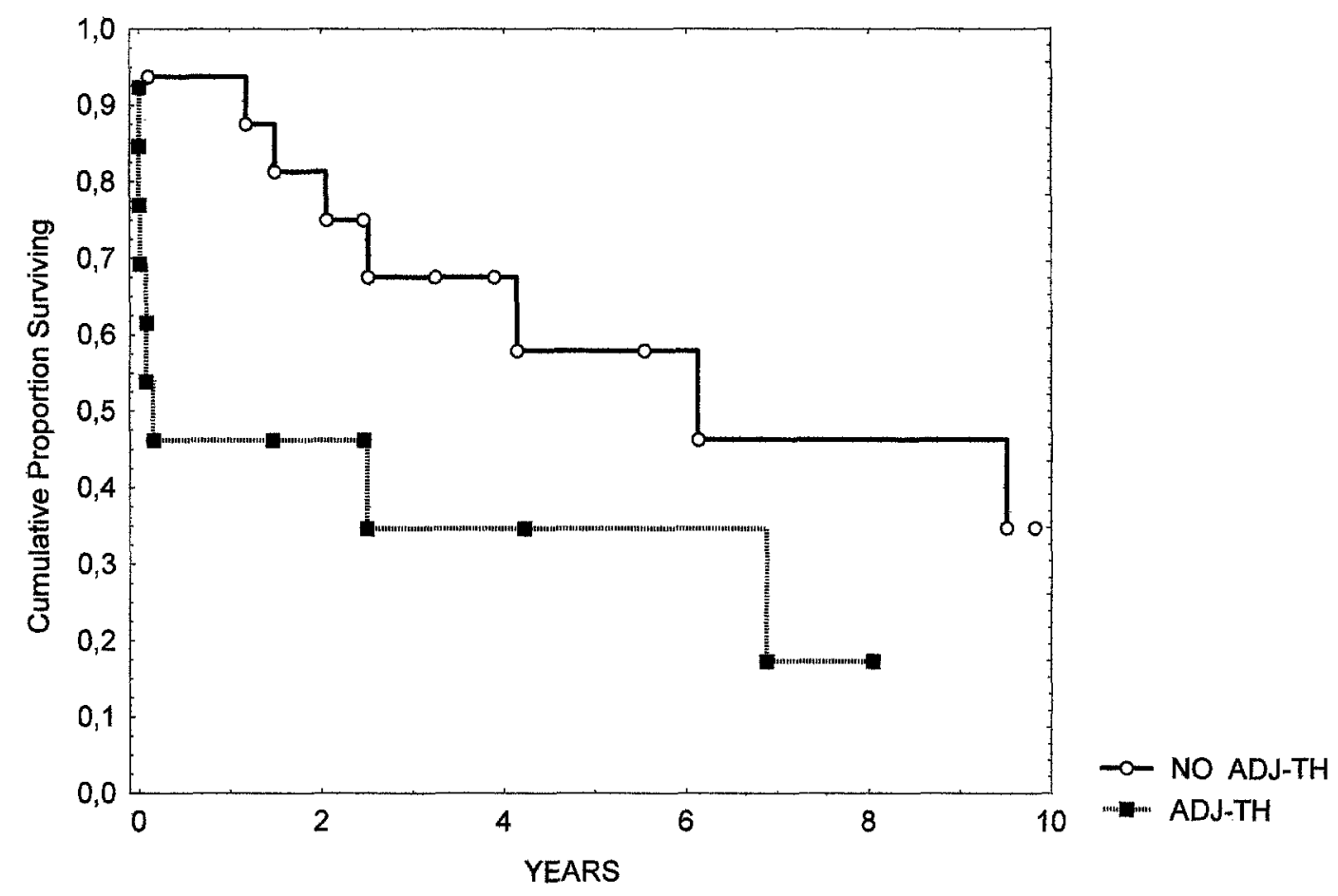

Fig. 4. Survival in cases of recurrent thymoma by the use of adjuvant radiotherapy $(A D J-T H)$ after the resection of initial thymoma; $p=0.04$.

extensively investigated. Some authors found that myasthenic thymomas recurred less frequently than nonmyasthenic thymomas. ${ }^{2,3}$ By contrast, other studies reported a modest and not significant difference in the recurrence rates between the two groups. ${ }^{6}$ In the present study we did not find any significant difference in the incidence of recurrence, extent of the recurrence, or survival between MG and non-MG recurrent thymomas.

The disease-free interval from the initial resection to recurrence has been reported to be as long as 10 years ${ }^{6,7}$ and longer after resection of encapsulated thymomas. Our results confirm these findings. We agree, therefore, that lifetime surveillance and careful follow-up of patients who undergo resection of thymoma (either encapsulated or invasive) are strongly advocated. In addition, we did not find an association between a longer disease-free interval and a better survival rate either by univariate or multivariate analysis. Recurrence after resection of the initial thymoma may be local or distant. In our population, a local recurrence confined to the mediastinum without pleural dissemination occurred in $39 \%(11 / 28)$ of the cases, whereas intrathoracic or extrathoracic dissemination (both considered as distant recurrence) were present in the remaining $61 \%$
$(17 / 28)$. We, in accordance with findings of other authors, ${ }^{9}$ observed that local and distant recurrences occur with similar frequencies for each stage of the initial thymoma, irrespective of the presence of associated MG disease. A significantly better survival is expected in the presence of local recurrence with a 5-year survival approximating that of the population of patients with resected nonrecurrent thymomas. Importantly, the extent of the recurrence was a significant prognostic factor in a multivariate survival analysis.

Optimal treatment of the recurrent tumor may be exceedingly difficult because of the general technical difficulties of second operation, the frequent involvement of intrathoracic organs, the possibility of intrathoracic dissemination of the recurrence, and the presence of associated severe myasthenic conditions. The two main therapeutic options, radiotherapy and operation, present advantages and disadvantages and have been widely used by a number of authors both separately and combined with conflicting results. Unfortunately, only a few series are available in the literature that compare patients receiving operation with patients receiving radiotherapy for treatment of the recurrent tumor. ${ }^{14}$ In a previous report, we indicated that better survival 


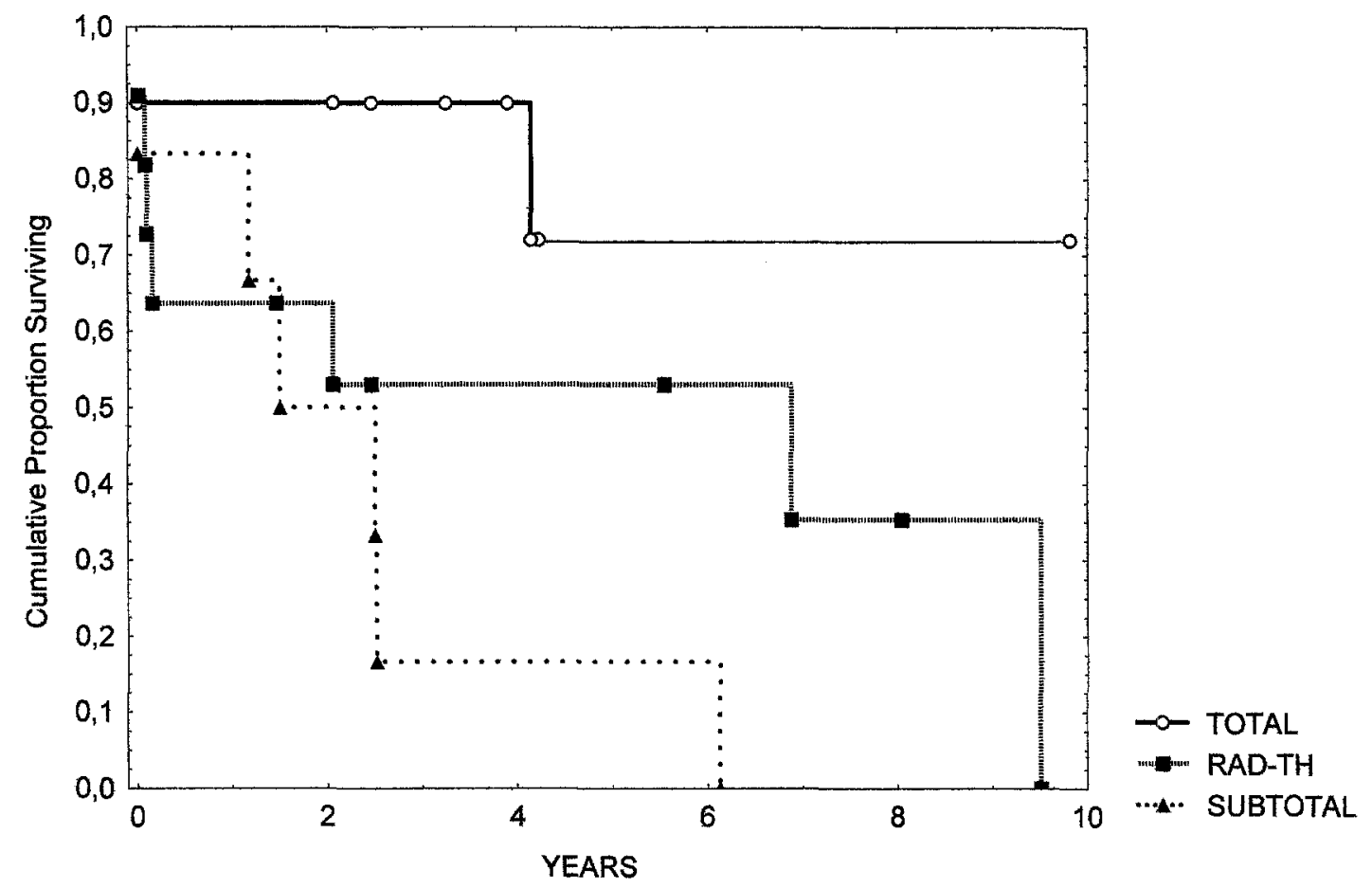

Fig. 5. Survival by the treatment of the recurrence. Total, Total resection; $R A D-T H$, exclusive radiotherapy; Subtotal, subtotal resection; $p=0.008$ total versus subtotal plus exclusive radiotherapy.

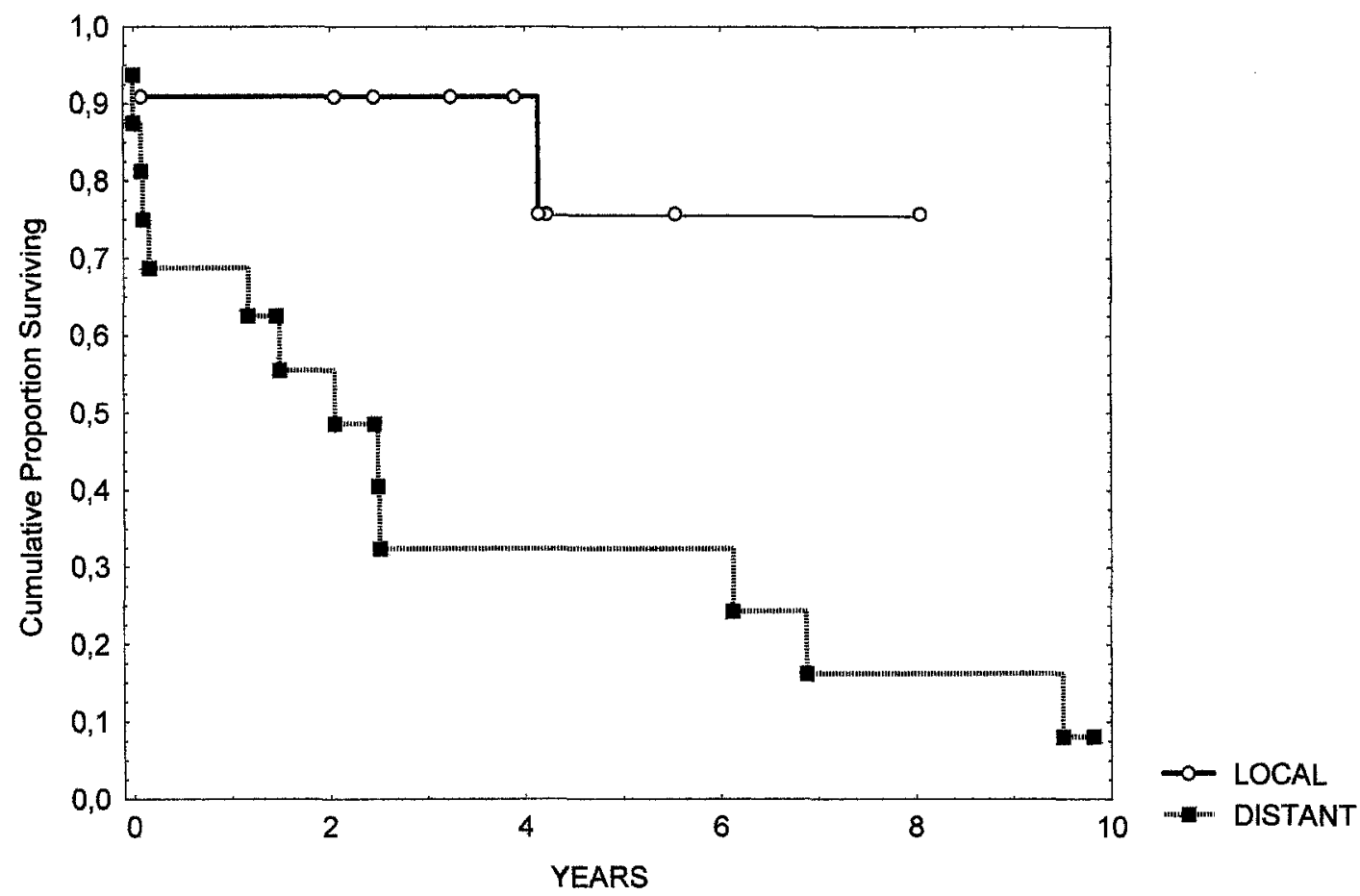

Fig. 6. Survival by the extent of recurrence. Local, Local recurrence; Distant, distant recurrence; $p=$ 0.004 . 
was expected when tumor recurrence was surgically treated than when nonsurgical therapies (radiotherapy with or without chemotherapy) were used, but at that time the difference was not significant. ${ }^{8}$ An additional study from our department on 21 patients with intrathoracic recurrence showed a 7-year survival of $74 \%$ when operation was used and of $65 \%$ when only radiotherapy was used; again, the difference was not significant. ${ }^{15}$ The results of the present study on a larger number of patients suggest that total resection of the recurrent tumor offers the best chance of long-term survival. A subtotal resection, often expression of more extensive disease, is usually associated with a poor prognosis, even with the addition of radiation therapy. It should be noted, however, that the patients were not randomly recruited for the treatment of the recurrence; after a careful preoperative and radiologic study those patients with a presumably resectable recurrent tumor were offered surgical operation, whereas those patients with a supposedly more advanced recurrence were given radiation therapy. This ethically unavoidable clinical bias implies that the difference of survival curves may result from a different condition of the recurrent tumor rather than from an actual effect of therapy. Despite the limits of a retrospective analysis, however, we recommend operation whenever feasible in recurrent thymomas, and similar conclusions have been reached by other authors. ${ }^{16}$ Complete resection of the recurrent tumor is a formidable task for the surgeon, because of the presence of post-actinic fibrous tissue, neoplastic invasion of mediastinal structures, and scar tissue from the initial operation. In the present series, a total resection of the recurrent tumor was possible in $62 \%(10 / 16)$ of the cases in which operation was done $(10 / 30,33 \%$ of the entire series), and 5-year survival in this group was excellent $(72 \%)$. The inability to perform a radical resection often results from involvement of the heart, ascending aorta, or main pulmonary artery. In most cases, this may only be ascertained at the time of operation.

Adjuvant radiotherapy after resection of thymoma has been found in several studies to reduce recurrence rates, to increase the disease-free interval to recurrence, and to improve long-term survival after the development of the recurrence..$^{3,17,18}$ By contrast, some authors have reached the opposite conclusion. ${ }^{19,20}$ In the present study, adjuvant radiotherapy after the initial resection was not associated with a reduction of the incidence of recurrence; it had no effect on prolonging the recurrence inter- val and did not increase survival after the development of the recurrence. Our results are in accordance with those of other investigators who found that overall recurrence rates of invasive thymomas were not significantly decreased by postoperative mediastinal irradiation., ${ }^{90,21}$ The results of the effect of adjuvant radiotherapy on recurrence must be interpreted with great caution because, at least in the present study, the allocation of patients with invasive thymomas to the radiation or no-radiation group after the initial resection was not random. Of 114 patients with invasive thymomas, 29 received radiotherapy after operation and 85 did not. The selection of patients might well have been biased, and it is possible that patients who received radiotherapy were selected because of a more clinically advanced stage, even if they had received a total resection. This selection bias can account for the surprising survival advantage in the no-radiation group, which was evident in both univariate and multivariate analysis. Nonetheless, even when this consideration is taken into account, one might hypothesize that the beneficial effect of postoperative radiotherapy on recurrence is questionable and deserves further investigation.

In conclusion, the major message of the present study on 30 recurrent thymomas out of 266 total resections is that patients with local recurrence in whom a total resection of the recurrent tumor may be accomplished have an excellent prognosis. Survival of these patients is similar to that of patients with complete resection in whom recurrence did not develop.

\section{REFERENCES}

1. Cohen DJ, Ronnigen LD, Graeber GM, et al. Management of patients with malignant thymoma. $J$ Thorac Cardiovasc Surg 1984;87:301-7.

2. Nakahara K, Ohno K, Hashimoto J, et al. Thymoma: results with complete resection and adjuvant postoperative irradiation in 141 consecutive patients. J Thorac Cardiovasc Surg 1988;95:1041-7.

3. Monden Y, Nakahara K, Iioka S, et al. Recurrence of thymoma: clinicopathological features, therapy and prognosis. Ann Thorac Surg 1985;39:165-9.

4. Marks RD Jr, Wallace KM, Pettit HS. Radiation therapy control of nine patients with malignant thymoma. Cancer 1978;41:117-9.

5. Rea F, Sartori F, Loy M, et al. Chemotherapy and operation for invasive thymoma. J Thorac Cardiovasc Surg 1993;106: 543-9.

6. Lewis JE, Wick MR, Scheithauer BW, Bernatz PE, Taylor WF. Thymoma: a clinicopathologic review. Cancer 1987;60: 2727-43.

7. Verley JM, Hollmann KH. Thymoma: a comparative study of 
clinical stages, histologic features, and survival in 200 cases. Cancer 1985;55:1074-86.

8. Maggi G, Casadio C, Cavallo A, Cianci R, Molinatti M, Ruffini E. Thymoma: results of 241 operated cases. Ann Thorac Surg 1991;51:152-6.

9. Haniuda M, Morimoto M, Nishimura H, Kobayashi O, Yamanda T, lida F. Adjuvant radiotherapy after complete resection of thymoma. Ann Thorac Surg 1992;54:311-5.

10. Masaoka A, Monden Y, Nakahara K, Tanioka T. Follow-up study of thymomas with special reference to their clinical stages. Cancer 1981;48:2485-92.

11. Lee ET. Statistical methods for survival data analysis. 2 nd ed. New York: John Wiley, 1992:243-80.

12. Shamji F, Pearson FG, Todd TRJ, Ginsberg RJ, Ilves R, Cooper JD. Results of surgical treatment for thymoma. J Thorac Cardiovase Surg 1984;87:43-7.

13. Fujimura S, Kondo T, Handa M, Shiraishi Y, Tamahashi N, Nakada T. Results of surgical treatment for thymoma based on 66 patients. J Thorac Cardiovasc Surg 1987;93:708-14.

14. Ribet M, Voisin C, Pruvot FR, Ramon P, Dambron P. Lymphoepithelial thymomas: a retrospective study of 88 resections. Eur J Cardiothorac Surg 1988;2:261-4.
15. Urgesi A, Monetti U, Rossi G, Ricardi U, Maggi G, Sannazzari GL. Aggressive treatment of intrathoracic recurrences of thymoma. Radiother Oncol 1992;24:221-5.

16. Kirschner PA. Reoperation for thymoma: report of 23 cases. Ann Thorac Surg 1990;49:550-5.

17. Batata MA, Martini N, Huvos AG, Aguilar RI, Beattie EJ Jr. Thymomas: clinicopathologic features, therapy and prognosis. Cancer 1974;34:389-96.

18. Simpson WJ. Radiotherapy of thymic tumors. In: Givel J-C, ed. Surgery of the thymus: pathology, associated disorders and surgical technique. Berlin: Springer-Verlag, 1990:299306.

19. Slater G, Papatestas AE, Genkins G, Kornfeld P, Horowitz $\mathrm{SH}$, Bender A. Thymomas in patients with myasthenia gravis. Ann Surg 1978;188:171-4.

20. Blumberg D, Port JL, Weksler B, et al. Thymoma: a multivariate analysis of factors predicting survival. Ann Thorac Surg 1995;60:908-14.

21. Crucitti F, Doglietto GB, Bellantone R, Perri V, Tommasini $O$, Tonali $P$. Effects of surgical treatment in thymoma with myasthenia gravis: our experience in 103 patients. J Surg Oncol 1992;50:43-6. 